Supporting Information for

\title{
SIGNAL-3L 3.0: IMPROVING SIGNAL PEPTIDE PREDICTION \\ THROUGH COMBINING ATTENTION DEEP LEARNING WITH WINDOW-BASED SCORING
}

\author{
Wei-Xun Zhang, Xiaoyong Pan, and Hong-Bin Shen
}

Institute of Image Processing and Pattern Recognition, Shanghai Jiao Tong University, and Key

Laboratory of System Control and Information Processing, Ministry of Education of China, Shanghai, 200240, China

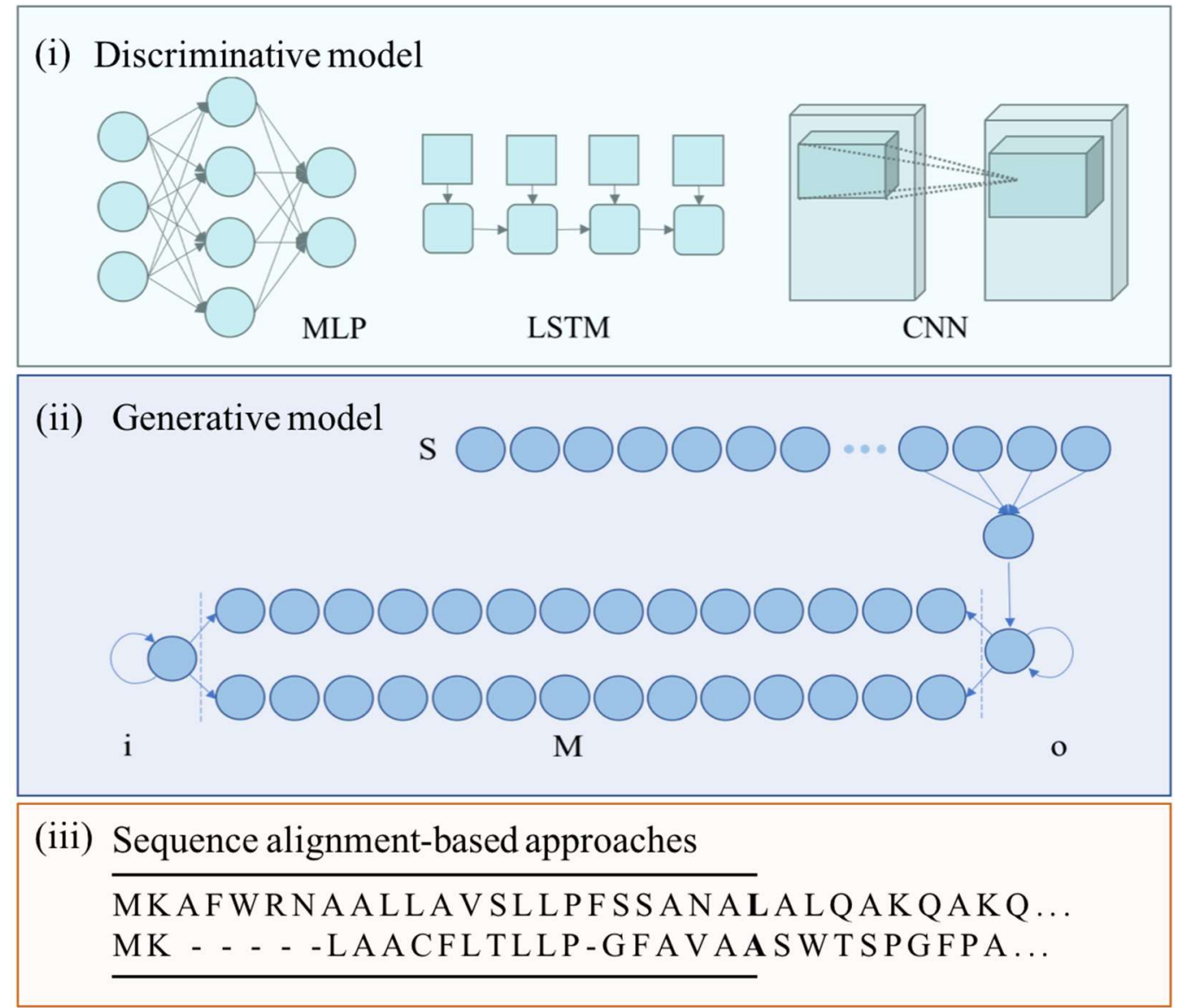

Figure S1. Existing prediction protocols of three different types of signal peptide predictors: (i) methods based on discriminative models, which inputs each residue feature (represented by a numeric feature vector) to generate a probability score; (ii) methods adopted Hidden Markov model to represent three regions $(\mathrm{N}-, \mathrm{H}-$, and $\mathrm{C}-$ regions) of signal peptide and gives a region-based estimation. (iii) sequence alignment based methods try to transfer the annotation from the annotated database according to evolutionary conservation. 


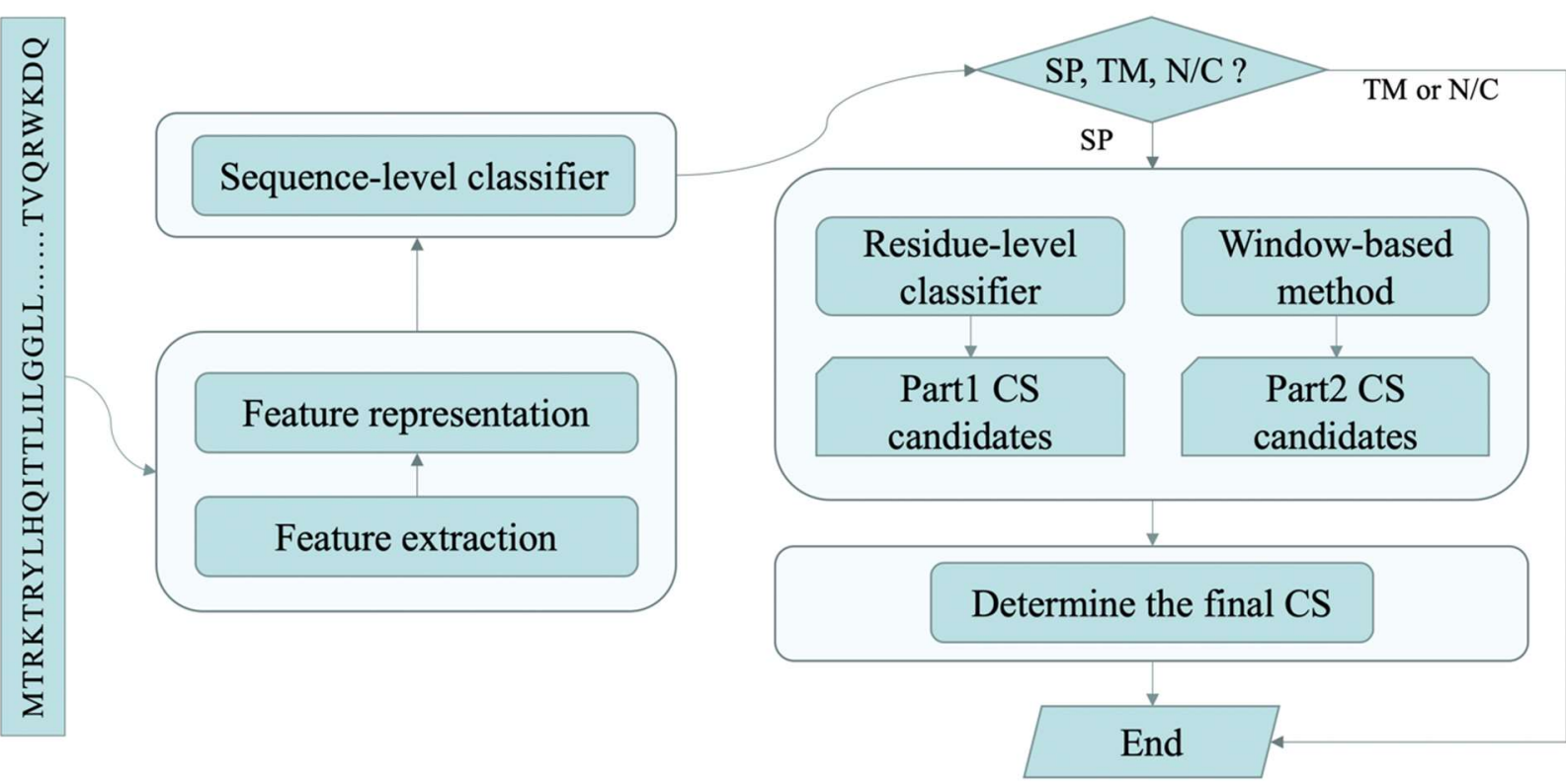

Figure S2. The overall prediction protocol of Signal-3L 3.0. First, we use programs (BLAST and HHblits) to encode each residue into a 50-dimensional vector. Then, a sequence-level classifier is used to judge whether the protein sequence contains signal peptide, transmembrane helix or is a globular protein. If the sequence is predicted to contain no signal peptide, the procedure is terminated. Otherwise, a residual-level classifier and a window-based method further generate the candidate cleavage sites, and the score of these two parts of candidate cleavage sites is weighted and fused to determine the final unique cleavage site. SP refers to signal peptide; TM refers to transmembrane helix; N/C refers to nuclear or cytosolic globular proteins; CS refers to cleavage site.

\section{Window-based cleavage site scoring}

The window-based scoring method uses a sliding window $-\xi_{1}, \ldots,-3,-2,-1,+1,+2, \ldots,+\xi_{2}$ along the protein sequence. Some amino acids occur more frequently on the three positions $(-3,-1,+1)$ when the cleavage site is located between -1 and +1 . The probability of the true cleavage site between -1 and +1 within the $k$ th sliding window with $\xi_{1}=13$ and $\xi_{2}=2$ is calculated as below:

$$
\begin{gathered}
\theta_{k}=p^{+}\left(R_{-13}\right) \ldots p^{+}\left(R_{-3}\right) p^{+}\left(R_{-2}\right) p^{+}\left(R_{-1} \mid R_{-3}\right) p^{+}\left(R_{+1} \mid R_{-1}\right) p^{+}\left(R_{+2}\right) \\
-p^{-}\left(R_{-13}\right) \ldots p^{-}\left(R_{-3}\right) p^{-}\left(R_{-2}\right) p^{-}\left(R_{-1} \mid R_{-3}\right) p^{-}\left(R_{+1} \mid R_{-1}\right) p^{-}\left(R_{+2}\right)
\end{gathered}
$$

where $p^{+}\left(R_{i}\right)$ and $p^{-}\left(R_{i}\right)$ respectively correspond to the probability of residue $R$ appearing at the $i$ th position in the positive and negative datasets, respectively, and $p\left(R_{i} \mid R_{i}\right)$ is the conditional probability.

\section{Model optimization}

The nested 5-fold cross-validation is used to train and evaluate Signal-3L 3.0. In SignalP 5.0 dataset, the benchmark (Bench) dataset is a subset of the training data. When performing the nested 5-fold cross-validation procedure, the SignalP 5.0 dataset is randomly divided into five subsets. During each fold of nested 5-fold cross-validation, one is selected as the test set (contains one fifth of the Bench dataset), and the remaining 4 subsets are used to train four models. After completing 
the 4-fold cross-validation of the internal loop, four models are obtained, and the average predicted results of these 4 models on this test set are obtained. Since the Bench dataset is a subset of the SignalP 5.0 dataset, some samples in this test set also belong to the Bench dataset. We pick out the prediction results of these shared samples in the Bench set. The above process is repeated 5 times, then the prediction results of Signal-3L 3.0 for the whole Bench dataset are obtained. In total, we obtain 20 models, which are used for webserver and predictions for SP19 dataset. The whole process is illustrated in Figure S3.

\section{Firstly: Split into five subsets}

Train Set:

\section{Secondly: Nested cross-validation procedure}

\begin{tabular}{|c|c|c|c|c|}
\hline Train & Train & Train & Valid & Test \\
\hline
\end{tabular}

Figure S3. The process of nested cross-validation.

The hyperparameters, e.g. learning rate $(0.1,0.01,0.001$, and 0.0001$)$, the number of Bi-LSTM layers $(1,2,3)$ and hidden neurons $(64,128,256)$, the number of $1 \mathrm{D}-\mathrm{CNN}$ layers $(1,2,3)$ and convolutional kernel size $(2,3,4,5)$, the number of neurons in the fully connected layer $(64$, $128,256)$, are optimized using cross-validation. The loss functions of the sequence-level classifier model and the residue-level classifier model are both cross entropy. Early-stopping is applied for model training. The early stop condition of the sequence-level classifier model is that the MCC score on the validation set for continuous multiple rounds does not increase. The early stop condition of the residue-level classifier model is that the signal peptide cleavage site recognition rate on the validation set for continuous multiple rounds does not increase.

In the nested 5-fold cross-validation, corresponding to the final 20 models, 20 scaling factor $\alpha$ are calculated. In Signal-3L 3.0, all the Bi-LSTM layers have 256 hidden neurons. In the sequencelevel classifier, both two linear projection layers have a hidden size of 512, the three fully connected layers have a hidden size of 512, 512 and 3, respectively. In the residue-level classifier, the firstlayer 1-D CNN has 64 feature maps with a kernel size of 3, the second-layer 1D-CNN has 32 feature maps with a convolution kernel size of 5. The two fully connected layers have a hidden size of 160 and 64, respectively. All codes were implemented using Pytorch version 1.1.0.

\section{Performance evaluation metrics}

$$
\begin{gathered}
\text { Recall }=\frac{T P}{T P+F N} \\
\text { Precision }=\frac{T P}{T P+F P} \\
F 1-\text { score }=2 \times \frac{\text { Precision } \times \text { Recall }}{\text { Precision }+} \\
M C C=\frac{T P \cdot T N-F N \cdot F P}{\sqrt{(T P+F N)(T P+F P)(T N+F N)(T N+F P)}}
\end{gathered}
$$


In SP prediction, we define proteins containing signal peptides as positive samples and transmembrane helices and other non-secretory proteins as negative samples. In cleavage site prediction, we define the protein sequence correctly predicted to contain signal peptide in SP prediction and correctly identified the cleavage site as TP. The exact match between the predicted and true positions of cleavage site is denoted as the distance 0 according to SignalP 5.0 definition. Similarly, the performance for $\pm 1, \pm 2, \pm 3$ nearby the true cleavage sites is also calculated, which considers the prediction is correct if the distance between the position of the predicted cleavage site and the position of the true cleavage site is less than or equal to 1,2 , and 3 , respectively.

Table S1. Details of the SignalP 5.0 benchmark dataset used in this study ${ }^{\text {a }}$

\begin{tabular}{lllllllll}
\hline & \multicolumn{2}{c}{ SP } & \multicolumn{2}{c}{ TM } & \multicolumn{2}{c}{ N/C } & \multicolumn{2}{c}{ Total } \\
\cline { 2 - 9 } Organism & Train & Bench & Train & Bench & Train & Bench & Train & Bench \\
\hline Euk & 2614 & 210 & 1044 & 318 & 13612 & 6929 & 17270 & 7457 \\
GP & 509 & 90 & 220 & 50 & 202 & 103 & 931 & 243 \\
GN & 189 & 25 & 50 & 25 & 140 & 64 & 379 & 114 \\
\hline
\end{tabular}

${ }^{a}$ Euk: Eukaryotes; GP: Gram-positive; GN: Gram-negative; SP: proteins with signal peptide; TM: transmembrane-helical proteins; $\mathrm{N} / \mathrm{C}=$ Nuclear and/or Cytosolic proteins; Bench: benchmark set.

Table S2. Details of the SP19 independent dataset in this study ${ }^{\mathrm{a}}$.

\begin{tabular}{llll}
\hline Organism & Eukaryotes & Gram-positive & Gram-negative \\
\hline Raw (Test) & $67(33)$ & $9(9)$ & $5(3)$ \\
\hline
\end{tabular}

a Raw represents the number of newly annotated SP proteins from Uniprot Knowledgebase released from 2018_04 to 2019_07. Test represents the remaining proteins in Raw after removing redundancy against SignalP 5.0 dataset and the internal redundancy.

Table S3. Cross-validation performance comparison of one vs. two or three LSTM layers for signal peptide recognition measured by MCC.

\begin{tabular}{cccc}
\hline Number of & Eukaryotes & Gram-negative & Gram-positive \\
\cline { 2 - 4 } LSTM layers & MCC & MCC & MCC \\
\hline One & 0.984 & 0.985 & 0.979 \\
Two & 0.980 & 0.978 & 0.968 \\
Three & 0.978 & 0.976 & 0.960 \\
\hline
\end{tabular}

Table S4. Confusion matrices for the different type of predictions that Signal-3L 3.0 makes on the benchmark dataset.

\begin{tabular}{c|c|c|c|c|c|c|c|c|c}
\hline & \multicolumn{3}{|c|}{ Eukaryotes } & \multicolumn{3}{c|}{ Gram-negative } & \multicolumn{3}{c}{ Gram-positive } \\
\hline real/predicted & SP & TM & CN & SP & TM & CN & SP & TM & CN \\
\hline SP & 198 & 4 & 8 & 87 & 3 & 0 & 24 & 1 & 0 \\
\hline TM & 7 & 290 & 21 & 0 & 49 & 1 & 0 & 24 & 1 \\
\hline CN & 12 & 21 & 6896 & 1 & 3 & 99 & 0 & 2 & 62 \\
\hline
\end{tabular}


Table S5. Signal peptide recognition measured by MCC, the MCCs of other methods are directly from SignalP 5.0.

\begin{tabular}{l|c|c|c}
\hline \multirow{2}{*}{ Method } & Eukaryotes & Gram-negative & Gram-positive \\
\cline { 2 - 4 } & MCC & MCC & MCC \\
\hline Signal-3L 3.0 & 0.925 & 0.965 & 0.974 \\
\hline SignalP 5.0 & 0.883 & 0.860 & 0.922 \\
\hline SignalP4.1 & 0.808 & 0.851 & 0.949 \\
\hline DeepSig & 0.819 & 0.792 & 0.870 \\
\hline Signal-3L 2.0 & 0.597 & 0.806 & 0.922 \\
\hline LipoP & 0.363 & 0.787 & 0.922 \\
\hline Philius & 0.421 & 0.802 & 0.843 \\
\hline Phobius & 0.510 & 0.818 & 0.818 \\
\hline PolyPhobius & 0.456 & 0.844 & 0.852 \\
\hline PrediSi & 0.553 & 0.802 & 0.781 \\
\hline PRED-LIPO & 0.234 & 0.775 & 0.896 \\
\hline PRED-SIGNAL & 0.272 & 0.724 & 0.830 \\
\hline PRED-TAT & 0.326 & 0.769 & 0.558 \\
\hline Signal-CF & 0.326 & 0.561 & 0.722 \\
\hline SOSUIsignal & 0.375 & 0.693 & 0.646 \\
\hline SPEPlip & 0.655 & 0.746 & 0.922 \\
\hline SPOCTOPUS & 0.492 & 0.860 & 0.897 \\
\hline TOPCONS2 & 0.477 & 0.860 &
\end{tabular}

Table S6. Signal peptide cleavage site prediction measured by recall on SignalP 5.0 dataset. The results of other methods are directly from SignalP 5.0.

\begin{tabular}{l|c|c|c|c|c|c|c|c|c|c|c|c}
\hline \multirow{2}{*}{ Method } & \multicolumn{3}{l}{ Eukaryotes } & \multicolumn{4}{l}{ Gram-negative } & \multicolumn{3}{l}{ Gram-positive } \\
\cline { 2 - 15 } & 0 & \pm 1 & \pm 2 & \pm 3 & 0 & \pm 1 & \pm 2 & \pm 3 & 0 & \pm 1 & \pm 2 & \pm 3 \\
\hline Signal-3L 3.0 & 0.676 & 0.752 & 0.800 & 0.829 & 0.667 & 0.722 & 0.789 & 0.822 & 0.760 & 0.800 & 0.880 & 0.880 \\
\hline SignalP 5.0 & 0.729 & 0.762 & 0.795 & 0.833 & 0.733 & 0.767 & 0.800 & 0.800 & 0.840 & 0.840 & 0.880 & 0.880 \\
\hline SignalP 4.1 & 0.695 & 0.729 & 0.762 & 0.786 & 0.644 & 0.711 & 0.733 & 0.744 & 0.840 & 0.840 & 0.840 & 0.840 \\
\hline DeepSig & 0.624 & 0.652 & 0.690 & 0.724 & 0.600 & 0.656 & 0.667 & 0.678 & 0.760 & 0.760 & 0.840 & 0.840 \\
\hline Signal-3L 2.0 & 0.648 & 0.686 & 0.733 & 0.762 & 0.644 & 0.700 & 0.722 & 0.733 & 0.800 & 0.800 & 0.840 & 0.840 \\
\hline LipoP & 0.343 & 0.386 & 0.419 & 0.448 & 0.733 & 0.767 & 0.789 & 0.789 & 0.600 & 0.600 & 0.640 & 0.640 \\
\hline Philius & 0.619 & 0.686 & 0.743 & 0.781 & 0.700 & 0.744 & 0.789 & 0.811 & 0.600 & 0.600 & 0.600 & 0.600 \\
\hline Phobius & 0.667 & 0.700 & 0.738 & 0.786 & 0.644 & 0.722 & 0.789 & 0.811 & 0.600 & 0.600 & 0.600 & 0.600 \\
\hline PolyPhobius & 0.681 & 0.733 & 0.776 & 0.833 & 0.644 & 0.733 & 0.811 & 0.822 & 0.680 & 0.680 & 0.720 & 0.720 \\
\hline PrediSi & 0.652 & 0.695 & 0.719 & 0.767 & 0.722 & 0.789 & 0.811 & 0.822 & 0.640 & 0.640 & 0.760 & 0.800 \\
\hline PRED-LIPO & 0.095 & 0.114 & 0.152 & 0.181 & 0.467 & 0.522 & 0.567 & 0.600 & 0.760 & 0.760 & 0.760 & 0.760 \\
\hline PRED-SIGNAL & 0.224 & 0.290 & 0.329 & 0.362 & 0.444 & 0.522 & 0.622 & 0.644 & 0.680 & 0.680 & 0.720 & 0.720 \\
\hline PRED-TAT & 0.410 & 0.510 & 0.571 & 0.614 & 0.711 & 0.767 & 0.800 & 0.822 & 0.720 & 0.720 & 0.760 & 0.760 \\
\hline Signal-CF & 0.652 & 0.676 & 0.724 & 0.762 & 0.689 & 0.711 & 0.744 & 0.778 & 0.720 & 0.720 & 0.800 & 0.800 \\
\hline SOSUIsignal & 0.176 & 0.329 & 0.467 & 0.576 & 0.267 & 0.367 & 0.567 & 0.622 & 0.200 & 0.240 & 0.280 & 0.440 \\
\hline
\end{tabular}




\begin{tabular}{l|l|l|l|l|l|l|l|l|l|l|l|l}
\hline SPEPlip & 0.710 & 0.733 & 0.771 & 0.810 & 0.611 & 0.678 & 0.722 & 0.733 & 0.680 & 0.680 & 0.720 & 0.720 \\
\hline SPOCTOPUS & 0.390 & 0.533 & 0.686 & 0.757 & 0.467 & 0.689 & 0.833 & 0.867 & 0.640 & 0.760 & 0.800 & 0.880 \\
\hline TOPCONS2 & 0.371 & 0.505 & 0.638 & 0.729 & 0.544 & 0.622 & 0.733 & 0.767 & 0.240 & 0.320 & 0.400 & 0.440 \\
\hline
\end{tabular}

Table S7. Signal peptide cleavage site prediction measured by precision on SignalP 5.0 dataset. The results of other methods are directly from SignalP 5.0.

\begin{tabular}{l|c|c|c|c}
\hline \multirow{2}{*}{ Method } & \multicolumn{4}{c}{ Eukaryotes } \\
\cline { 2 - 5 } & 0 & \pm 1 & \pm 2 & \pm 3 \\
\hline Signal-3L 3.0 & 0.654 & 0.728 & 0.774 & 0.802 \\
\hline SignalP 5.0 & 0.671 & 0.702 & 0.732 & 0.732 \\
\hline SignalP4.1 & 0.613 & 0.643 & 0.672 & 0.693 \\
\hline DeepSig & 0.604 & 0.631 & 0.668 & 0.700 \\
\hline Signal-3L 2.0 & 0.322 & 0.341 & 0.365 & 0.379 \\
\hline LipoP & 0.159 & 0.178 & 0.194 & 0.207 \\
\hline Philius & 0.151 & 0.168 & 0.182 & 0.191 \\
\hline Phobius & 0.226 & 0.237 & 0.250 & 0.267 \\
\hline PolyPhobius & 0.176 & 0.190 & 0.201 & 0.216 \\
\hline PrediSi & 0.273 & 0.291 & 0.301 & 0.321 \\
\hline PRED-LIPO & 0.069 & 0.083 & 0.110 & 0.131 \\
\hline PRED-SIGNAL & 0.066 & 0.085 & 0.096 & 0.106 \\
\hline PRED-TAT & 0.08 & 0.099 & 0.111 & 0.119 \\
\hline Signal-CF & 0.105 & 0.109 & 0.117 & 0.123 \\
\hline SOSUIsignal & 0.037 & 0.069 & 0.098 & 0.121 \\
\hline SPEPlip & 0.366 & 0.378 & 0.398 & 0.418 \\
\hline SPOCTOPUS & 0.120 & 0.164 & 0.211 & 0.233 \\
\hline TOPCONS2 & 0.107 & 0.146 & 0.184 & 0.210 \\
\hline
\end{tabular}

Table S8. Benchmarking of signal peptide cleavage site prediction measured by F1-score on SignalP 5.0 dataset. The results of other methods are directly from SignalP 5.0.

\begin{tabular}{l|c|c|c|c}
\hline \multirow{2}{*}{ Method } & \multicolumn{4}{c}{ Eukaryotes } \\
\cline { 2 - 5 } & 0 & \pm 1 & \pm 2 & \pm 3 \\
\hline Signal-3L 3.0 & 0.665 & 0.740 & 0.787 & 0.815 \\
\hline SignalP 5.0 & 0.699 & 0.731 & 0.762 & 0.779 \\
\hline SignalP4.1 & 0.651 & 0.683 & 0.714 & 0.737 \\
\hline DeepSig & 0.614 & 0.641 & 0.679 & 0.712 \\
\hline Signal-3L 2.0 & 0.430 & 0.456 & 0.487 & 0.506 \\
\hline LipoP & 0.217 & 0.244 & 0.265 & 0.283 \\
\hline Philius & 0.243 & 0.270 & 0.292 & 0.307 \\
\hline Phobius & 0.338 & 0.354 & 0.373 & 0.399 \\
\hline PolyPhobius & 0.280 & 0.302 & 0.319 & 0.343 \\
\hline PrediSi & 0.385 & 0.410 & 0.424 & 0.453 \\
\hline PRED-LIPO & 0.080 & 0.096 & 0.128 & 0.152 \\
\hline
\end{tabular}




\begin{tabular}{l|l|l|l|l}
\hline PRED-SIGNAL & 0.102 & 0.131 & 0.149 & 0.164 \\
\hline PRED-TAT & 0.134 & 0.166 & 0.186 & 0.199 \\
\hline Signal-CF & 0.181 & 0.188 & 0.201 & 0.212 \\
\hline SOSUIsignal & 0.061 & 0.114 & 0.162 & 0.200 \\
\hline SPEPlip & 0.483 & 0.499 & 0.525 & 0.551 \\
\hline SPOCTOPUS & 0.184 & 0.251 & 0.323 & 0.356 \\
\hline TOPCONS2 & 0.166 & 0.227 & 0.286 & 0.326 \\
\hline
\end{tabular}

Table S9. Benchmarking of signal peptide recognition and its cleavage site prediction on SP19 independent dataset. (ID 1-33 are Eukaryotic sequences, 34-36 are Gram-negative sequences, 3745 are Gram-positive sequences. Column Name records the name of the protein sequence. Column $G T$ indicates the experimental label of the signal peptide cleavage site annotated in the database. $P_{Y}$ represents the recognition result of the method on whether the sequence contains signal peptide, $\mathrm{Y}$ is inclusive, $\mathrm{N}$ is exclusive. $P_{c s}$ represents the result of the method for predicting signal peptide cleavage site.

\begin{tabular}{|c|c|c|c|c|c|c|c|c|c|c|c|c|c|c|}
\hline & \multicolumn{2}{|c|}{ Signal proteins } & \multicolumn{2}{|c|}{ DeepSig } & \multicolumn{2}{|c|}{ Phobius } & \multicolumn{2}{|c|}{ PRED-TAT } & \multicolumn{2}{|c|}{ PrediSi } & \multicolumn{2}{|c|}{ SignalP 5.0} & \multicolumn{2}{|c|}{ Signal-3L 3.0 } \\
\hline ID & Name & $G T$ & $P_{Y}$ & $P_{c S}$ & $P_{Y}$ & $P_{c s}$ & $P_{Y}$ & $P_{C S}$ & $P_{Y}$ & $P_{c S}$ & $P_{Y}$ & $P_{c s}$ & $P_{Y}$ & $P_{c S}$ \\
\hline 1 & AMP1_PARCM & 23 & $\mathrm{Y}$ & 23 & $\mathrm{Y}$ & 23 & $\mathrm{Y}$ & 23 & $\mathrm{~N}$ & - & $\mathrm{Y}$ & 23 & $\mathrm{Y}$ & 23 \\
\hline 2 & AMP2_PARCM & 23 & $\mathrm{Y}$ & 23 & $\mathrm{Y}$ & 23 & $\mathrm{Y}$ & 21 & $\mathrm{Y}$ & 23 & $\mathrm{Y}$ & 23 & $\mathrm{Y}$ & 23 \\
\hline 3 & AMP3_PARCM & 23 & $\mathrm{Y}$ & 23 & $\mathrm{Y}$ & 23 & $\mathrm{Y}$ & 23 & $\mathrm{Y}$ & 23 & $\mathrm{Y}$ & 23 & $\mathrm{Y}$ & 23 \\
\hline 4 & AMPU1_AMPCP & 26 & $\mathrm{Y}$ & 24 & $\mathrm{Y}$ & 24 & $\mathrm{Y}$ & 24 & $\mathrm{~N}$ & - & $\mathrm{Y}$ & 24 & $\mathrm{Y}$ & 24 \\
\hline 5 & AMPU2_AMPCP & 26 & $\mathrm{Y}$ & 24 & $\mathrm{Y}$ & 24 & $\mathrm{Y}$ & 24 & $\mathrm{~N}$ & - & $\mathrm{Y}$ & 24 & $\mathrm{Y}$ & 24 \\
\hline 6 & AMPU3_AMPCP & 17 & $\mathrm{~N}$ & - & $\mathrm{N}$ & - & $\mathrm{N}$ & - & $\mathrm{N}$ & - & $\mathrm{Y}$ & 20 & $\mathrm{Y}$ & 17 \\
\hline 7 & ANIS5_ANISI & 18 & $\mathrm{Y}$ & 18 & $\mathrm{Y}$ & 18 & $\mathrm{Y}$ & 18 & $\mathrm{Y}$ & 18 & $\mathrm{Y}$ & 18 & $\mathrm{Y}$ & 18 \\
\hline 8 & APIM_APIME & 24 & $\mathrm{~N}$ & - & $\mathrm{Y}$ & 24 & $\mathrm{Y}$ & 24 & $\mathrm{Y}$ & 24 & $\mathrm{Y}$ & 24 & $\mathrm{Y}$ & 24 \\
\hline 9 & CG2RA_CONMI & 29 & $\mathrm{Y}$ & 22 & $\mathrm{Y}$ & 19 & $\mathrm{Y}$ & 19 & $\mathrm{Y}$ & 19 & $\mathrm{Y}$ & 19 & $\mathrm{Y}$ & 22 \\
\hline 10 & CMU1_USTMA & 21 & $\mathrm{Y}$ & 21 & $\mathrm{Y}$ & 21 & $\mathrm{Y}$ & 22 & $\mathrm{Y}$ & 21 & $\mathrm{Y}$ & 21 & $\mathrm{Y}$ & 23 \\
\hline 11 & CROS1_CROSA & 26 & $\mathrm{Y}$ & 25 & $\mathrm{Y}$ & 25 & $\mathrm{Y}$ & 25 & $\mathrm{Y}$ & 25 & $\mathrm{Y}$ & 25 & $\mathrm{Y}$ & 25 \\
\hline 12 & DEP23_DERPT & 21 & $\mathrm{Y}$ & 21 & $\mathrm{Y}$ & 21 & $\mathrm{Y}$ & 21 & $\mathrm{Y}$ & 21 & $\mathrm{Y}$ & 21 & $\mathrm{Y}$ & 21 \\
\hline 13 & GH5FP_CHAOB & 28 & $\mathrm{~N}$ & - & $\mathrm{Y}$ & 28 & $\mathrm{Y}$ & 28 & $\mathrm{Y}$ & 28 & $\mathrm{Y}$ & 28 & $\mathrm{Y}$ & 26 \\
\hline 14 & IBPKA_TYPIS & 20 & $\mathrm{~N}$ & - & $\mathrm{Y}$ & 20 & $\mathrm{Y}$ & 20 & $\mathrm{Y}$ & 20 & $\mathrm{Y}$ & 20 & $\mathrm{Y}$ & 20 \\
\hline 15 & IVOB_EMENI & 18 & $\mathrm{Y}$ & 19 & $\mathrm{Y}$ & 19 & $\mathrm{Y}$ & 19 & $\mathrm{Y}$ & 19 & $\mathrm{Y}$ & 19 & $\mathrm{Y}$ & 19 \\
\hline 16 & PNX36_SCOSD & 25 & $\mathrm{Y}$ & 25 & $\mathrm{Y}$ & 30 & $\mathrm{Y}$ & 25 & $\mathrm{Y}$ & 25 & $\mathrm{Y}$ & 25 & $\mathrm{Y}$ & 25 \\
\hline 17 & SCX5B_MESEU & 19 & $\mathrm{Y}$ & 19 & $\mathrm{Y}$ & 19 & $\mathrm{Y}$ & 20 & $\mathrm{Y}$ & 19 & $\mathrm{Y}$ & 19 & $\mathrm{Y}$ & 19 \\
\hline 18 & THAP1_THAPI & 18 & $\mathrm{Y}$ & 16 & $\mathrm{Y}$ & 16 & $\mathrm{Y}$ & 16 & $\mathrm{Y}$ & 16 & $\mathrm{Y}$ & 16 & $\mathrm{Y}$ & 16 \\
\hline 19 & TX13A_MYRGU & 24 & $\mathrm{~N}$ & - & $\mathrm{Y}$ & 24 & $\mathrm{Y}$ & 24 & $\mathrm{Y}$ & 24 & $\mathrm{Y}$ & 24 & $\mathrm{Y}$ & 24 \\
\hline 20 & TX14A_MYRGU & 25 & $\mathrm{Y}$ & 25 & $\mathrm{Y}$ & 19 & $\mathrm{Y}$ & 23 & $\mathrm{~N}$ & - & $\mathrm{Y}$ & 25 & $\mathrm{Y}$ & 25 \\
\hline 21 & TX51A_SCOSD & 24 & $\mathrm{Y}$ & 24 & $\mathrm{Y}$ & 24 & $\mathrm{Y}$ & 24 & $\mathrm{Y}$ & 24 & $\mathrm{Y}$ & 24 & $\mathrm{Y}$ & 24 \\
\hline 22 & TXA1A_SCOSD & 23 & $\mathrm{Y}$ & 23 & $\mathrm{Y}$ & 23 & $\mathrm{Y}$ & 21 & $\mathrm{~N}$ & - & $\mathrm{Y}$ & 23 & $\mathrm{Y}$ & 23 \\
\hline 23 & TXA2A_SCOSD & 23 & $\mathrm{Y}$ & 23 & $\mathrm{Y}$ & 23 & $\mathrm{Y}$ & 20 & $\mathrm{~N}$ & - & $\mathrm{Y}$ & 23 & $\mathrm{Y}$ & 50 \\
\hline 24 & TXF1A_SCOMU & 23 & $\mathrm{Y}$ & 23 & $\mathrm{Y}$ & 22 & $\mathrm{Y}$ & 23 & $\mathrm{Y}$ & 23 & $\mathrm{Y}$ & 23 & $\mathrm{Y}$ & 23 \\
\hline 25 & TXF1A_SCOSD & 20 & $\mathrm{Y}$ & 20 & $\mathrm{Y}$ & 20 & Y & 20 & $\mathrm{Y}$ & 20 & Y & 20 & $\mathrm{Y}$ & 20 \\
\hline
\end{tabular}




\begin{tabular}{c|l|c|c|c|c|c|c|c|c|c|c|c|c|c}
\hline 26 & TXF2A_SCOSD & 20 & $\mathrm{Y}$ & 18 & $\mathrm{Y}$ & 19 & $\mathrm{~N}$ & - & $\mathrm{N}$ & - & $\mathrm{Y}$ & 20 & $\mathrm{Y}$ & 20 \\
\hline 27 & TXF3A_SCOSD & 23 & $\mathrm{Y}$ & 23 & $\mathrm{Y}$ & 22 & $\mathrm{Y}$ & 23 & $\mathrm{Y}$ & 23 & $\mathrm{Y}$ & 23 & $\mathrm{Y}$ & 23 \\
\hline 28 & TXF3B_SCOSD & 23 & $\mathrm{Y}$ & 23 & $\mathrm{Y}$ & 22 & $\mathrm{Y}$ & 23 & $\mathrm{Y}$ & 23 & $\mathrm{Y}$ & 23 & $\mathrm{Y}$ & 23 \\
\hline 29 & TXG1A_SCOSD & 23 & $\mathrm{Y}$ & 23 & $\mathrm{Y}$ & 26 & $\mathrm{Y}$ & 21 & $\mathrm{Y}$ & 23 & $\mathrm{Y}$ & 23 & $\mathrm{Y}$ & 23 \\
\hline 30 & TXX1A_ETHRU & 24 & $\mathrm{Y}$ & 24 & $\mathrm{Y}$ & 24 & $\mathrm{Y}$ & 22 & $\mathrm{Y}$ & 24 & $\mathrm{Y}$ & 24 & $\mathrm{Y}$ & 24 \\
\hline 31 & TXX1B_ETHRU & 24 & $\mathrm{Y}$ & 24 & $\mathrm{Y}$ & 24 & $\mathrm{Y}$ & 22 & $\mathrm{Y}$ & 24 & $\mathrm{Y}$ & 24 & $\mathrm{Y}$ & 24 \\
\hline 32 & XEG1_PHYSP & 19 & $\mathrm{Y}$ & 19 & $\mathrm{Y}$ & 19 & $\mathrm{Y}$ & 19 & $\mathrm{Y}$ & 19 & $\mathrm{Y}$ & 19 & $\mathrm{Y}$ & 19 \\
\hline 33 & ZONAD_KOMPC & 19 & $\mathrm{Y}$ & 19 & $\mathrm{Y}$ & 19 & $\mathrm{Y}$ & 19 & $\mathrm{Y}$ & 20 & $\mathrm{Y}$ & 19 & $\mathrm{Y}$ & 19 \\
\hline 34 & BPAC_BURP2 & 71 & $\mathrm{~N}$ & - & $\mathrm{N}$ & - & $\mathrm{N}$ & - & $\mathrm{Y}$ & 58 & $\mathrm{~N}$ & - & $\mathrm{Y}$ & 25 \\
\hline 35 & CDIA_ECONC & 32 & $\mathrm{Y}$ & 22 & $\mathrm{~N}$ & - & $\mathrm{Y}$ & 29 & $\mathrm{~N}$ & - & $\mathrm{Y}$ & 31 & $\mathrm{Y}$ & 30 \\
\hline 36 & MTO_HYPSQ & 24 & $\mathrm{Y}$ & 24 & $\mathrm{Y}$ & 24 & $\mathrm{Y}$ & 24 & $\mathrm{Y}$ & 24 & $\mathrm{Y}$ & 24 & $\mathrm{Y}$ & 24 \\
\hline 37 & CHPD_STRCO & 23 & $\mathrm{Y}$ & 30 & $\mathrm{Y}$ & 23 & $\mathrm{Y}$ & 23 & $\mathrm{~N}$ & - & $\mathrm{Y}$ & 23 & $\mathrm{Y}$ & 23 \\
\hline 38 & CHPE_STRCO & 27 & $\mathrm{Y}$ & 34 & $\mathrm{Y}$ & 27 & $\mathrm{Y}$ & 27 & $\mathrm{Y}$ & 27 & $\mathrm{Y}$ & 27 & $\mathrm{Y}$ & 27 \\
\hline 39 & CHPF_STRCO & 36 & $\mathrm{Y}$ & 42 & $\mathrm{Y}$ & 35 & $\mathrm{Y}$ & 35 & $\mathrm{Y}$ & 32 & $\mathrm{Y}$ & 35 & $\mathrm{Y}$ & 35 \\
\hline 40 & CHPG_STRCO & 27 & $\mathrm{Y}$ & 34 & $\mathrm{Y}$ & 37 & $\mathrm{Y}$ & 27 & $\mathrm{~N}$ & - & $\mathrm{Y}$ & 27 & $\mathrm{Y}$ & 31 \\
\hline 41 & CHPH_STRCO & 25 & $\mathrm{Y}$ & 31 & $\mathrm{Y}$ & 25 & $\mathrm{Y}$ & 25 & $\mathrm{Y}$ & 25 & $\mathrm{Y}$ & 25 & $\mathrm{Y}$ & 25 \\
\hline 42 & INLC_LISMG & 34 & $\mathrm{Y}$ & 34 & $\mathrm{Y}$ & 34 & $\mathrm{Y}$ & 34 & $\mathrm{Y}$ & 29 & $\mathrm{Y}$ & 34 & $\mathrm{Y}$ & 34 \\
\hline 43 & PSRP_STRPN & 72 & $\mathrm{~N}$ & - & $\mathrm{N}$ & - & $\mathrm{N}$ & - & $\mathrm{Y}$ & 34 & $\mathrm{~N}$ & - & $\mathrm{Y}$ & 34 \\
\hline 44 & RDLA_STRCO & 28 & $\mathrm{Y}$ & 28 & $\mathrm{Y}$ & 28 & $\mathrm{Y}$ & 22 & $\mathrm{Y}$ & 21 & $\mathrm{Y}$ & 28 & $\mathrm{Y}$ & 28 \\
\hline 45 & RDLB_STRCO & 28 & $\mathrm{Y}$ & 28 & $\mathrm{Y}$ & 22 & $\mathrm{Y}$ & 22 & $\mathrm{Y}$ & 20 & $\mathrm{Y}$ & 28 & $\mathrm{Y}$ & 28 \\
\hline
\end{tabular}

